\title{
Selective renal angiography in Wegener's granulomatosis
}

\author{
H. M. MOUTSOPOULOS, P. C. AVGERINOS, C. G. TSAMPOULAS, AND \\ P. A. KATSIOTIS
}

From the Departments of Medicine and Radiology, School of Medicine, University of Ioannina, Ioannina, Greece

SUMMARY Two patients whose illnesses meet the criteria of Wegener's granulomatosis are presented. Selective renal angiography performed in both revealed multiple arterial aneurysms similar to those classically found in polyarteritis nodosa.

In the last 15 years selective angiography, particularly of the kidney, has been proposed as a valuable diagnostic and follow-up procedure in polyarteritis nodosa. ${ }^{12}$ The characteristic angiographic appearance in polyarteritis nodosa consists of multiple small aneurysms that are uniform in size, 1 to $5 \mathrm{~mm}$, and associated with medium and small arteries exclusively. ${ }^{1-3}$

Selective renal angiography has not been used extensively in Wegener's granulomatosis, and sporadic reports have been negative ${ }^{45}$ with the exception of a single case whose angiographic changes were similar to those of polyarteritis nodosa. ${ }^{6}$

We present 2 patients with Wegener's granulomatosis. Selective renal angiography in both revealed multiple arterial aneurysms similar to those observed in polyarteritis nodosa.

\section{Case reports}

CASE 1

A 30-year-old machinist was admitted to our unit with a month's history of fever, frontal headache, cough, and nasal discharge. On examination he was febrile (temperature $39 \cdot 3^{\circ} \mathrm{C}$ ) and normotensive. Tenderness over frontal and maxillary sinuses was noted. Rales were heard on auscultation of his right lung base. Laboratory investigation showed the following abnormal results: haematocrit $38 \%$, total white blood count $9.8 \times 10^{9} / 1$ (polymorphonuclear cells $72 \%$, lymphocytes $26 \%$, monocytes $2 \%$ ), erythrocyte sedimentation rate was $116 \mathrm{~mm} / \mathrm{h}$. Urine Accepted for publication 16 March 1982.

Correspondence to $\mathrm{Dr} \mathrm{H}$. M. Moutsopoulos, Department of Medicine, School of Medicine, Ioannina, Greece. microscopy showed 10 red blood cells per highpower optic field. Sinus $x$-rays showed diffuse opacification of maxillary and left frontal sinuses. A chest $x$-ray revealed multiple, well-demarcated, nodular, cavitating lesions in both lung fields (Fig. 1). Biopsy of the nasal mucosa and the right maxillary sinus showed granulomatous necrotising vasculitis, consistent with Wegener's granulomatosis. The patient was started on a regimen of prednisone 1 $\mathrm{mg} / \mathrm{kg} /$ day and cyclophosphamide $2 \mathrm{mg} / \mathrm{kg} /$ day.

Bilateral selective renal angiography was performed 4 months after initiation of treatment. Kidneys were of normal size and shape. Multiple pinpoint microaneurysms and irregularities of the interlobular arteries were observed in both kidneys (Fig. 2).

CASE 2

A 53-year-old housewife was admitted with 20 days' history of fever, arthritis of the right wrist, arthralgia of both knees, and productive cough with blood stained sputum. On admission her temperature was $38.8^{\circ} \mathrm{C}$, blood pressure $120 / 80 \mathrm{mmHg}$, and pulse rate $120 / \mathrm{min}$. Mucosal ulcerations were noted on the hard palate, and rales were heard over the right lung base. Numerous ecchymoses and petechiae were observed, mainly over the lower limbs. Laboratory investigations gave the following abnormal results: haematocrit $30 \%$, total white blood cell count $14 \times 10^{9} / 1$ (polymorphonuclear cells $90 \%$, lymphocytes $8 \%$, monocytes $2 \%$ ). The erythrocyte sedimentation rate was $140 \mathrm{~mm} / \mathrm{h}$. Serum creatinine was $5.6 \mathrm{mg} / 100 \mathrm{ml}$ $(495 \mu \mathrm{mol} / \mathrm{l})$. Urine analysis revealed proteinuria of $1 \cdot 1 \mathrm{~g} / \mathrm{l}$ and specific gravity of 1008 . Urine microscopy showed numerous red and white blood cells and red blood cell casts. A large $(5 \times 3.5 \mathrm{~cm})$ cavitating 


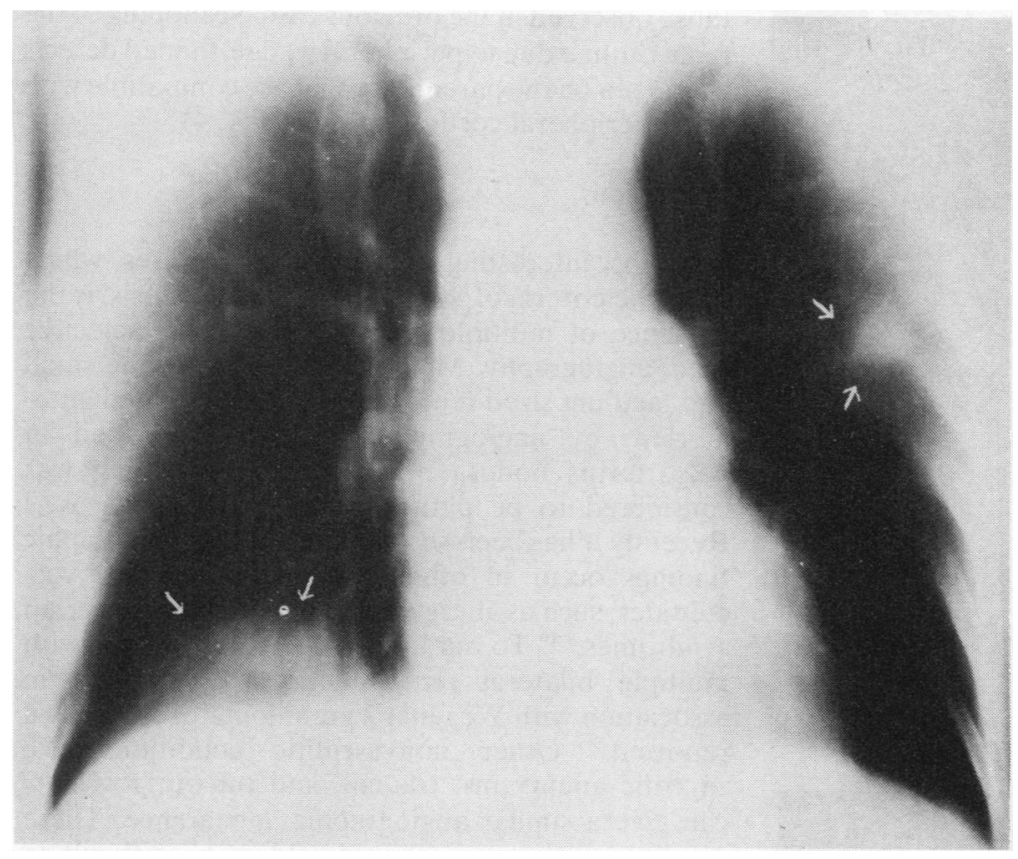

Fig. 1 Multiple cavitating pulmonary nodules shown by arrows.

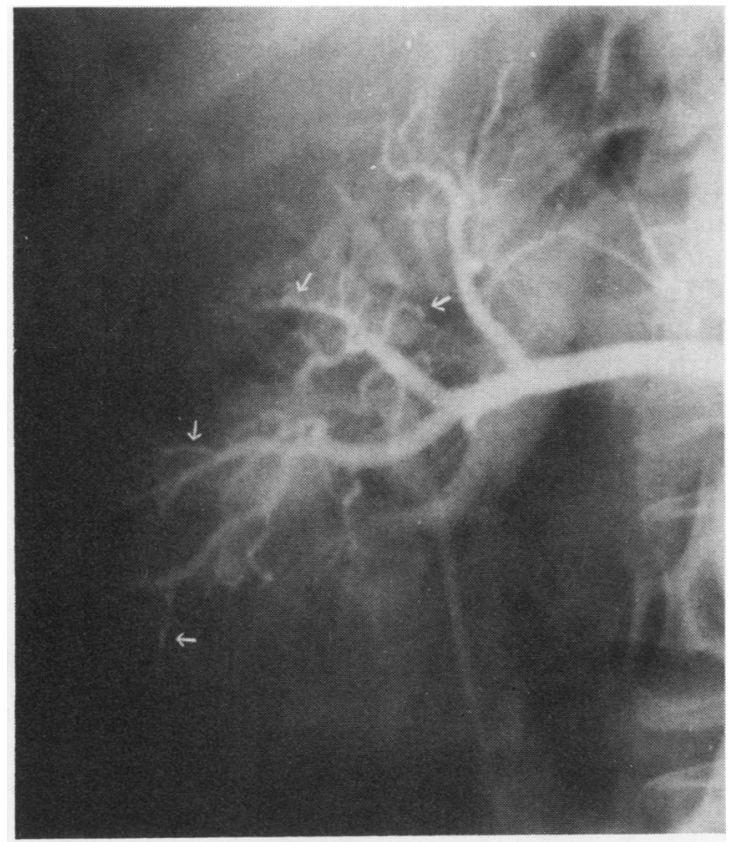

Fig. 2 Selective renal angiogram of the right kidney showing multiple pinpoint microaneurysms and irregularities of the interlobular arteries. Representative lesions are indicated by arrows. opacity in the right mid lung was seen on chest $x$-ray (Fig. 3). A purified protein derivative test, repeated twice, was negative. Multiple cultures of blood, sputum, urine, and right maxillary sinus aspirate were negative for common pathogens and Mycobacterium tuberculosis. Bone marrow, liver, and transbronchial lung biopsy showed normal appearances. Skull $x$-rays showed a uniform opacification of right maxillary sinus and a fluid level in the left maxillary sinus. A right maxillary sinus and nasal mucosal biopsy showed signs of chronic inflammation. Over the following days the serum creatinine rose to $14.2 \mathrm{mg} / 100$ $\mathrm{ml}(1255 \mu \mathrm{mol} / \mathrm{l})$ and she became anuric, requiring haemodialysis on alternate days.

A presumptive diagnosis of Wegener's granulomatosis was made on the basis of upper and lower respiratory tract involvement, cutaneous manifestations compatible with vasculitis, and clinical evidence of rapidly progressive glomerulonephritis. Diagnosis was further supported by an excellent response to treatment with cyclophosphamide (2 $\mathrm{mg} / \mathrm{kg} /$ day) and prednisone $(1 \mathrm{mg} / \mathrm{kg} /$ day $)$. She required only 4 haemodialysis sessions. After a month of treatment the creatinine returned to normal. She underwent a bilateral selective renal angiography. Both kidneys were of normal size. Interlobular arteries showed irregularities of lumen and multiple small aneurysms, which were larger than 


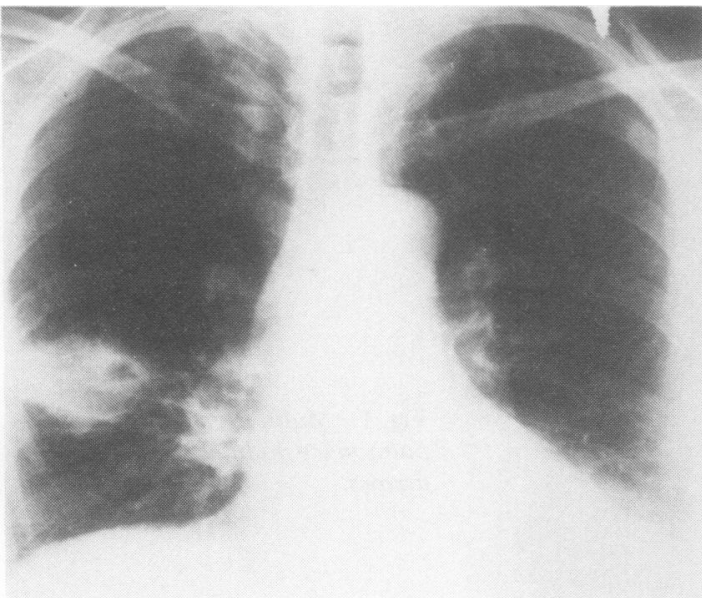

Fig. 3 Chext $\mathrm{x}$-ray showing a large $(5 \times 3.5 \mathrm{~cm})$ cavitating nodule in right mid-lung.

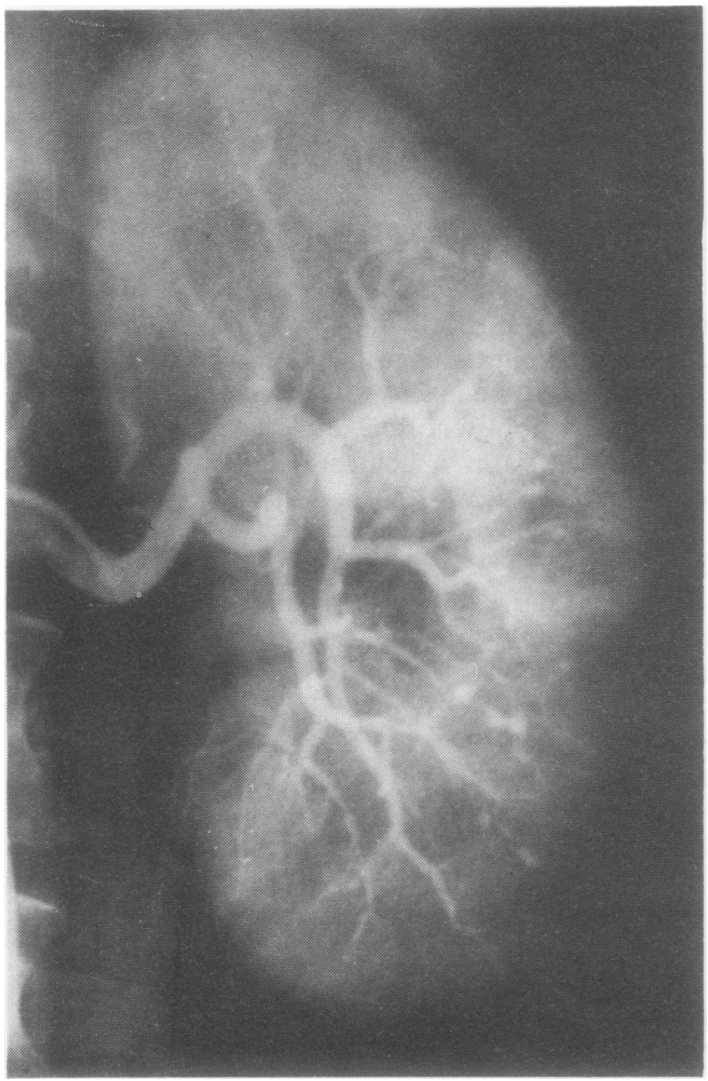

Fig. 4 Selective renal angiogram of left kidney showing multiple microaneurysms and scalloping of the renal outline. those observed in the previous case. Scalloping of the renal outline due to peripheral wedge-shaped defects were seen on the parenchymal phase compatible with small peripheral cortical infarcts (Fig. 4).

\section{Discussion}

The most interesting aspect of these 2 cases, which meet the criteria of Wegener's granulomatosis, is the presence of multiple microaneurysms on selective renal angiography. Multiple aneurysms of the small and medium sized renal arteries demonstrated antemortem by angiography was first described in polyarteritis nodosa in $1965 .{ }^{17}$ This finding was considered to be pathognomonic of the disease. ${ }^{8}$ Recently it has been shown that similar angiographic findings occur in other systemic necrotising vasculitides, such as allergic granulomatosis and overlap syndromes. ${ }^{910}$ To our knowledge only one case with multiple bilateral renal aneurysms occurring in association with Wegener's granulomatosis has been reported. ${ }^{6}$ Other nonvasculitic conditions like mycotic aneurysms, trauma, and tumour invasion ${ }^{1}$ can give a similar angiographic appearance. These conditions, however, should seldom be difficult to differentiate clinically from a necrotising vasculitis.

It is of interest that the angiographic findings appeared to parallel the severity of glomerular involvement. In the first case, in which microscopic haematuria was the only evidence of glomerular involvement, angiography showed relatively few pinpoint aneurysms. In the second case, however, where strong evidence of extensive glomerular damage existed, the aneurysms were larger, more numerous, and associated with wedge-shaped peripheral infarcts.

The 2 cases suggest that arterial aneurysms may be a more frequent finding in Wegener's granulomatosis than previously thought. ${ }^{4-6}$ It is also possible, if one considers the presence of arterial aneurysms as enough evidence for the diagnosis of an overlap syndrome, that these 2 cases represent an overlap syndrome between Wegener's granulomatosis and polyarteritis nodosa. Finally, one is faced with the question of whether these 2 entities may represent different expressions of the same vasculitic process that leads to multiple aneurysm formation. Systematic study by selective renal angiography of such cases may solve this question.

We thank Dr D. Emmanuel for his suggestions and comments and Ms E. Papanikolaou for excellent secretarial assistance.

\section{References}

1 Fleming $\mathbf{R}$ J, Stem L Z. Multiple intraparenchymal renal aneurysms in polyarteritis nodosa. Radiology 1965; 84: 100-3. 
2 Kohn B J, Stulberg H J. Angiographic diagnosis of polyarteritis nodosa. West $J$ Med 1971; 114: 40-2.

3 Evens R G, Dorby C A, Eckert J F. An exercise in radiologicpathologic correlation. Radiology 1968; 91: 1028-32.

4 Ekelund L. Lindholm T. Angiography in collagenous disease of the kidney. Acta Radiol (Diagn) (Stockh) 1974; 15: 413-22.

5 Lundstrom B, Lindqvist B, Solerbergh $\mathrm{H}$, et al. Nephroangiography in Wegener's granulomatosis. A comparison with panarteritis nodosa. Acta Radiol (Diagn) (Stockh) 1975; 16: 641-53.

6 Baker S B, Robinson D R. Unusual renal manifestations of Wegener's granulomatosis. Am J Med 1978; 64: 883-9.
7 Bron K M, Strott C A, Shapiro A P. The diagnostic value of angiographic observations in polyarteritis nodosa. Arch Intern Med 1965; 116: 450-3.

8 Essigner A, Bonard M. Panarteritis nodosa, an angiographic entity presentation of two cases. Br J Radiol 1971; 44: 184-7.

9 Fauci A S, Haynes B F, Katz P. The spectrum of vasculitis. Clinical, pathologic, immunologic and therapeutic considerations. Ann Intern Med 1978; 89: 660-76.

10 Kohler P F, Claman H N. Systemic vasculitis. In: Franklin E C, ed. Clinical Immunology Update. New York: ChurchillLivingstone, 1981: 293-319. 